\title{
Revista Electrónica del Lenguaje, VII: El papel de la gramática del texto en la enseñanza de lenguas
}

Fernández Martín, P. (coord.) (2020): Revista electrónica del lenguaje: El papel de la gramática del texto en la enseñanza de lenguas, 7. ISSN: 2387-1598. https://www.revistaelectronicalenguaje.c om/cfp-volumen-vii_el-papel-de-la-gramatica-del-texto-en-la-ensenanza-de-lenguas/

\section{Reseña de Daniel Moríñigo Rodríguez Universidad de Alcalá}

La Revista Electrónica del Lenguaje es una publicación anual de acceso libre, cuyo fin es proporcionar un espacio de difusión para la labor investigadora de todo tipo de investigadores, sean docentes o estudiantes, sobre el área del lenguaje, desde una perspectiva que incorpora múltiples disciplinas. Así, en ella se admiten trabajos que versan desde aspectos concretos de la lengua, como la gramática o la lexicología, hasta la didáctica de primeras y segundas lenguas. Bajo esta última temática, se publica el número séptimo en 2020 , el cual trata de manera concreta el papel de la gramática del texto en la enseñanza de lenguas.

El número siete de la Revista cuenta con cinco secciones. La primera es una presentación redactada por la coordinadora del número: la Dra. Patricia Fernández, de la Universidad Autónoma de Madrid. El segundo apartado, es el "monográfico", donde se recogen artículos principalmente relacionados con el aspecto discursivo de las lenguas. La tercera sección, "miscelánea", incluye artículos de temática variada, como estudios de lingüística comparada o una investigación que analiza cuentos infantiles a través de la perspectiva de género. En la cuarta sección, dedicada a las "notas", podemos encontrar materiales útiles tanto para la investigación sobre el discurso como para la enseñanza de español. Finalmente, la quinta sección se dedica a reseñas de libros.

La presentación de Patricia Fernández nos proporciona un panorama general del número, con una descripción de su estructura y un resumen del contenido. La presentación proporciona una lectura amena y, al mismo tiempo, imprescindible para el lector que desee obtener un adelanto de la temática de las investigaciones, así como de sus conclusiones y aspectos más importantes. Tras esta síntesis, Patricia Fernández concluye con los oportunos agradecimientos y expresa, además, su esperanza de que el número sea una aportación útil en el ámbito de las lenguas y de la enseñanza. En las últimas líneas hay un recuerdo a quienes han sufrido por la pandemia, consciente de que la lengua no permite expresar la compasión que 
sentimos como nos gustaría. Como lectores, nos unimos en el sentimiento, agradeciendo asimismo que, en medio de circunstancias tan difíciles, se haya podido publicar este número, que podemos disfrutar de manera libre y gratuita.

En el MONOGRÁFICO, que conforma la primera sección, encontramos cuatro artículos que se centran, sobre todo, en cuestiones relativas a la gramática del texto. Julián Muñoz Pérez presenta, en el primer artículo, cinco secuencias didácticas "que pueden emplearse para potenciar el desarrollo de la competencia discursiva" (p. 10) y estudia su inclusión en varios manuales de enseñanza de español. La propuesta original del autor de los cinco modelos de secuencias didácticas puede ser bastante útil para el investigador que desee contar con una herramienta para analizar secuencias en manuales o para el profesor que luche con la planificación de sus propias clases. El artículo llega a una interesante conclusión: los manuales de español evidencian un fuerte predominio de la secuencia "presentación-prácticaproducción", centrada fundamentalmente en el nivel de la oración. Frente a esta tendencia, Julián Muñoz Pérez sugiere optar por otros modelos que le den más importancia al texto y que sean sensibles a "los factores cognitivos y afectivos" (p. 10).

El segundo artículo del monográfico de Cristina Corral Esteve examina la manera en que se enfocan, denominan y enseñan los marcadores discursivos en los manuales de enseñanza de español como lengua extranjera. En él, Cristina Corral se pregunta si esa forma de abordar los marcadores facilita su aprendizaje. Tras analizar un conjunto de manuales de la colección Vente (niveles A1 a B2), identifica varias carencias en este sentido. En primer lugar, los marcadores se suelen abordar desde una perspectiva fundamentalmente gramatical. En segundo lugar, no se enseñan utilizando una terminología clara. En tercer lugar, aunque de los niveles A1 a B1 se observa un incremento en la dificultad y cantidad de los marcadores enseñados, en el nivel B2 se observa una reducción injustificada, más teniendo en cuenta que en este nivel los alumnos trabajan con textos más complejos. En definitiva, Cristina Corral sugiere que se trabaje más con los marcadores en los manuales y que se haga desde una perspectiva pragmático-discursiva, dado que su sentido en los textos no siempre viene determinado por factores gramaticales.

Respecto a las críticas de Cristina Corral dirigidas a la carencia de una terminología clara sobre los marcadores discursivos, nos gustaría decir que esta puede deberse, sobre todo, a la necesidad de hacer estas unidades más accesibles a los alumnos en el contexto de su nivel de lengua y progresión. No obstante, es cierto que, desde determinado punto de vista, se podría 
argumentar que una terminología precisa, unificada y clara en diversos manuales (sobre todo si son de la misma editorial) podría facilitar su identificación y asimilación a largo plazo.

Considerando, esta vez, otra lengua, el tercer artículo del monográfico trata la traducción de los marcadores discursivos del español al alemán. Elke Cases Berbel parte de la premisa de que a los marcadores discursivos no se les presta la atención necesaria en los manuales de alemán como lengua extranjera, tesis similar a la sostenida en el artículo anterior, aunque aplicada a los manuales de ELE. Esta deficiencia en el abordaje de los marcadores tiene un efecto negativo sobre la naturalidad con la que los aprendientes deben construir sus textos en la lengua meta. Para solventar esta dificultad, Cases diseña un ejercicio de traducción español-alemán de acuerdo con la metodología del aprendizaje basado en problemas. El ejercicio consiste en la traducción de tres conectores de tipo aditivo, consecutivo y contraargumentativo. A la luz de los resultados observados tras el ejercicio, se concluye que un ejercicio de traducción como este ayuda a los aprendientes no solo a ser conscientes de la importancia de los conectores dentro del texto, sino a buscar equivalencias de conectores más que una traducción literal. Quizá la contribución más importante de este artículo sea la idea de que la traducción, un recurso que durante mucho tiempo y desde determinadas metodologías se ha dejado a un lado en favor de estrategias más intralingüísticas, es efectivamente útil a la hora de mejorar en los aprendientes el reconocimiento y uso adecuado de los marcadores discursivos.

El último artículo del monográfico resalta la necesidad de trabajar los mecanismos referenciales responsables de la cohesión y la coherencia de los textos, examinando esta vez el uso que los alumnos checos de español hacen de estos mecanismos al escribir. En un estudio que analiza dos corpus de textos, cada uno correspondiente a aprendientes con niveles de lengua diferentes, Jana Pešková observa que los alumnos transfieren procedimientos anafóricos de su lengua materna, como el hecho de no emplear demostrativos (este, ese y aquel). Además, incluso cuando su nivel de lengua aumenta, tienden a conformarse con los mismos recursos anafóricos de nivel básico, como, por ejemplo, el uso de pronombres personales ('él', 'ella'), el uso de posesivos ('su') o la repetición total ('Guillermo', 'María'). En definitiva, se vuelve a detectar una importante necesidad de dedicar tiempo a la enseñanza y a la práctica de estos mecanismos lingüísticos de carácter textual, así como de añadir mecanismos más avanzados según se progrese en el dominio de la lengua.

La sección de MISCELÁNEA abre con un artículo dedicado a la comparación de formas verbales que expresan la modalidad en español y polaco. Al tratarse de sistemas verbales que 
no son equivalentes, los alumnos polacos pueden encontrarse con dificultades a la hora de emplear verbos y estructuras de tipo modal. A través de un análisis comparativo de dos verbos modales y dos perífrasis verbales en español y polaco, Maciej Jaskot y Agniezska Wiltos llegan a la conclusión de que no basta con las correspondencias temporales entre las formas en ambos idiomas, ya que el español tiene un mayor número de tiempos verbales en pasado. Además, en algunos casos ciertas formas verbales en pasado requieren un cambio de modo verbal en polaco. Ante esta problemática, es imprescindible llevar a cabo una comparativa formal a la vez que pragmática. En el aula se podrían seleccionar casos problemáticos y aportar ejemplos de uso comparado, si bien es evidente que no siempre es posible ni merece la pena enredarse en disquisiciones metalingüísticas (pp. 125-126).

Nuevamente, es sorprendente comprobar cómo la solución a un problema de adquisición, en este caso, relacionado con las formas verbales que expresan el modo y con otras diferencias de significado, pasa por utilizar estrategias translingüísticas, frente a la tendencia intralingüística predominante en las últimas décadas. Se trata, pues, de una vuelta a la comparación, del mismo modo que en el artículo de Elke Cases Berbel hablábamos de una vuelta a la traducción. Independientemente de cuál sea nuestra postura en este sentido, no deja de ser interesante considerar información como la planteada por Maciej Jaskot y Agniezska Wiltos. Sin embargo, junto con las observaciones derivadas de este estudio, surge la necesidad de comprobar qué estrategias y técnicas concretas serían las más apropiadas para solventar las dificultades en el uso de formas verbales que expresan modo.

El segundo artículo de la sección miscelánea nos lleva una vez más al ámbito de la traducción y la lingüística contrastiva, esta vez comparando el español con el armenio. M. ${ }^{a}$ Azucena Penas Ibáñez y Nelli Minasyan ponen su atención en las traducciones de perífrasis modales de infinitivo y perífrasis aspectuales, buscando descubrir "el grado de convergencia semántica, léxica y [...] gramatical" (p. 130) entre español y armenio. Los diferentes ejemplos considerados en cuanto a las perífrasis modales de infinitivo revelan distintos grados de convergencia en la traducción. En algunos casos, existe una convergencia en los tres ámbitos (poder + infinitivo); en otros, solo una divergencia de tipo gramatical (deber + infinitivo y deber de + infinitivo); y, en otros, divergencia semántica, léxica y gramatical (haber de + infinitivo, haber que + infinitivo, tener que + infinitivo). De hecho, dentro de esta última circunstancia, existen casos como el de haber de + infinitivo en el que, en armenio, la construcción no puede ser perifrástica, dado que el verboide se sustituye por una oración introducida por 'que'. En segundo lugar, en la traducción de perífrasis aspectuales del español al armenio se pueden 
apreciar paralelismos formales con lenguas como el indoeuropeo, el griego y el latín. Finalmente, las autoras dedican un pequeño apartado en su artículo a estudiar las "construcciones de infinitivo flexionado en subordinadas completivas y adverbiales del portugués y su traducción al armenio, dado que en esta lengua también existe flexión morfemática de infinitivo" (p. 146). Tras el análisis de algunos ejemplos, se concluye que, a pesar de esta similitud a primera vista, no son iguales, particularmente debido al uso en portugués de la flexión desinencial de persona-número frente a la "flexión casual según la función sintáctica oracional del infinitivo en su dimensión nominal" (p. 150).

El artículo de M. ${ }^{a}$ Azucena Penas Ibáñez y Nelli Minasyan interesa de manera especial a investigadores que trabajen en las perífrasis en español o en el análisis contrastivo, ya sea entre español y armenio, portugués y armenio, o lenguas románicas y lenguas no románicas en general. Asimismo, algunas de las conclusiones podrían serle útiles al profesor de español que enseñe a alumnos cuya lengua materna sea el armenio, ya que ser consciente de las diferencias (semánticas, léxicas y gramaticales) en la manera en la que se traducirían las perífrasis, podrá facilitar la comprensión del profesor de sus propios alumnos y de las dificultades particulares que pudieran evidenciar por interferencia con su lengua materna.

El tercer artículo de esta sección de miscelánea utiliza la perspectiva de género como marco interpretativo para analizar cuentos infantiles que se utilizan en la enseñanza de inglés. $\mathrm{Su}$ autora, María Raposo Jiménez, se propone examinar la manera en la que se representan los personajes masculinos y femeninos en la literatura infantil. Aplicando una metodología que combina el análisis cuantitativo con el cualitativo, se estudian doce cuentos infantiles. Para el análisis cuantitativo, se utiliza un cuestionario que permite examinar el sexismo en contextos educativos, atendiendo a cuatro bloques de análisis: 1) presencia versus ausencia, 2) vida laboral versus vida familiar, 3) rol activo versus rol pasivo, 4) y estereotipos o roles representados (p. 164). Para el análisis cualitativo, se realiza un análisis de los símbolos asociados a cada género, sean de tipo visual (ilustraciones) o textual. La conclusión más importante es que sigue habiendo diferencias entre la representación de personajes femeninos y masculinos en la literatura infantil, a pesar de que se puede percibir cierto progreso en comparación con investigaciones previas. Lo interesante de este artículo es que el estudio se centra en el componente ideológico de los textos, de manera que el análisis transciende el nivel meramente gramatical, o incluso el pragmático-discursivo.

Pasando ya a las NOTAS, nos encontramos una primera titulada "Sobre el tratamiento discursivo de la inmigración en la prensa" de Susana Ridao Rodrigo, en la que, de modo 
similar al artículo anterior de María Raposo, asume la posición de analizar un conjunto de textos atendiendo a los discursos ideológicos que transmiten. Susana Ridao Rodrigo proporciona un marco teórico fundamentado en el análisis del discurso, junto con la sugerencia de emplearlo para examinar las ideologías que se transmiten desde los medios de comunicación con respecto a los movimientos migratorios. Por otro lado, también se proporciona un corpus que recoge las opiniones de alumnos de posgrado, de diferente procedencia, sobre la manera en que los medios representan los fenómenos de inmigración y emigración. Aunque el artículo no proporciona un estudio que analice y sintetice, en realidad, los discursos encontrados en los medios de comunicación o las opiniones expresadas por los alumnos de máster, sí proporciona tanto las ideas como los materiales para llevar a cabo una investigación como esta.

La última de las notas consiste en una unidad didáctica para la enseñanza de español, diseñada por Elena Benítez Rodríguez para un grupo pequeño de alumnos angloparlantes de educación infantil, con un nivel inicial absoluto de español. Basándose en la temática de la Navidad, sigue un enfoque multisensorial que procura hacer uso de medios auditivos, visuales, físicos y motrices; utilizar el español como lengua vehicular; tener en cuenta el componente afectivo y motivacional; y trabajar asimismo diversas destrezas. En este último sentido, se procura trabajar principalmente la expresión y comprensión oral; sin embargo, también se dedica una pequeña porción a la comprensión escrita, a través de la lectura simple de tarjetas (flashcards), así como a la expresión escrita, que se trabaja en la sesión final, en la que los aprendientes escribirían una carta a los Reyes Magos. Lo más útil de la unidad tal y como se encuentra en el artículo es que es bastante completa, pues no solo proporciona una sólida fundamentación teórica, sino que también describe todo lo que tiene que ver con el contexto de enseñanza, los contenidos, los objetivos, los materiales, la temporalización, las sesiones, y las actividades que se realizarían en cada una de ellas. Independientemente de si un profesor deseara emplearla tal cual o si pensara en adaptarla de alguna forma, parece claro que es una aportación relevante para el profesor de español que trabaje con niños de infantil. Por otro lado, puede ser de interés para los investigadores que quieran examinar aplicaciones prácticas de enfoques multisensoriales.

Finalmente, la última sección está dedicada a RESEÑAS de libros relacionados con diversos aspectos de la enseñanza y aprendizaje de lenguas, a saber: la enseñanza de unidades fraseológicas; la enseñanza de inglés en la Educación Secundaria; el desarrollo de la conciencia lingüística en los aprendientes de español como lengua materna y como lengua extranjera; el 
lenguaje corporal y los gestos de los españoles; la enseñanza de segundas y primeras lenguas en infantil y primaria; la enseñanza de español en primaria; el uso del miniensayo en la enseñanza de la escritura; y el desarrollo de las habilidades lingüísticas en la educación infantil. La peculiaridad de los libros reseñados es que no solo han sido redactados por "auténticos referentes en el área" (p. 7), como reconoce Patricia Fernández en la introducción de la revista, sino que, tal y como se puede entrever a través de la lectura de las reseñas, proporcionan una información completa teórica y práctica para los desafíos que los profesores de lenguas, maternas o extranjeras, pueden enfrentar en nuestros días. Por ello, es más que probable que los investigadores y docentes que deseen enriquecer sus perspectivas sobre la enseñanza de lenguas encuentren en esta sección una herramienta útil para conocer qué se está escribiendo actualmente. También, podrán encontrar títulos para incorporar a sus lecturas, según sus inquietudes y proyectos futuros.

En síntesis, el número séptimo de la Revista electrónica del lenguaje recoge investigaciones que consideran el apartado discursivo desde diferentes perspectivas, ofreciendo un amplio y enriquecedor conjunto de planteamientos, fundamentos teóricos, metodologías de investigación, conclusiones, ideas, recursos y herramientas. En algunos casos, el enfoque de los artículos recae sobre elementos lingüísticos específicos que contribuyen a la coherencia y cohesión de los textos, y que, por lo tanto, contribuyen al desarrollo de la competencia discursiva, como los marcadores discursivos y los recursos anafóricos. En este sentido, se examina desde la forma en la que se secuencian y enseñan estas unidades, hasta la manera en la que los aprendientes las perciben; llegando incluso a reconsiderar la traducción como herramienta pedagógica. En otros casos, el enfoque es eminentemente comparativo, profundizando en la manera en la que lenguas diferentes construyen estructuras determinadas. Por otro lado, también encontramos artículos que trascienden el nivel oracional o incluso pragmático para examinar las ideologías incrustadas en los textos, apoyándose en marcos analíticos como la perspectiva de género o el análisis del discurso. No podían faltar, tampoco, los materiales de carácter más práctico, donde encontramos desde una unidad didáctica plenamente desarrollada hasta la materia prima para llevar a cabo una investigación nueva. Si a todo esto le añadimos las reseñas que, como decíamos, pueden guiar la elección de lecturas relevantes, nos encontramos ante un número de gran utilidad debido a la variedad, rigurosidad y relevancia de sus contenidos. A todo esto, se le suma la ventaja de ser una publicación de acceso libre y gratuito. 